\title{
PROTOCOLO DE ENFERMAGEM PARA AS ALTERAÇÕES PSICOSSOCIAIS E ESPIRITUAIS DA PESSOA COM COLOSTOMIA*
}

\author{
Elaine Soares da Silva ${ }^{1}$, Denise Silveira de Castro², Walckiria Garcia Romero ${ }^{3}$, Telma Ribeiro Garcia ${ }^{4}$, Cândida Caniçali Primo ${ }^{5}$
}

${ }^{1}$ Enfermeira. Mestre em Enfermagem. Enfermeira da Secretaria de Saúde do Espírito Santo. Vitória, ES, Brasil.

${ }^{2}$ Enfermeira. Doutora em Enfermagem. Docente do Departamento de Enfermagem da Universidade Federal do Espírito Santo. Vitória, ES, Brasil.

${ }^{3}$ Enfermeira. Doutora em Fisiologia. Docente do Departamento de Enfermagem da Universidade Federal do Espírito Santo. Vitória, ES, Brasil.

${ }^{4}$ Enfermeira. Doutora em Enfermagem. Docente do Departamento de Enfermagem de Saúde Pública e Psiquiatria da Universidade Federal da Paraíba. João Pessoa, PB, Brasil.

${ }^{5}$ Enfermeira. Doutoranda em Enfermagem. Docente do Departamento de Enfermagem da Universidade Federal do Espírito Santo. Vitória, ES, Brasil.

RESUMO: Pesquisa exploratório-descritiva, realizada em 2013, com objetivo de elaborar um protocolo de assistência de enfermagem contendo os diagnósticos/resultados e intervenções de enfermagem, relacionados às necessidades psicossociais e psicoespirituais da pessoa com colostomia. Elaborou-se 47 diagnósticos/resultados e 57 intervenções de enfermagem utilizando a Classificação Internacional para a Prática de Enfermagem, organizados segundo o referencial teórico de Wanda de Aguiar Horta. Concluiu-se que a pessoa com colostomia se apresenta fragilizada e demanda cuidados de enfermagem específicos, individualizados, devido a aflições, medos e mudanças em seu estilo de vida. Esta classificação de enfermagem é de fácil utilização e a sua associação com as necessidades humanas básicas permitiu perceber a pessoa com colostomia de maneira integral, para além das técnicas e procedimentos de enfermagem.

DESCRITORES: Colostomia; Processos de enfermagem; Classificação; Terminologia; Teoria de enfermagem.

\section{NURSING PROTOCOL FOR PSYCHOSOCIAL AND SPIRITUAL ALTERATIONS OF THE PERSON WITH COLOSTOMY}

\section{PROTOCOLO DE ENFERMERÍA PARA LAS ALTERACIONES PSICOSOCIALES Y ESPIRITUALES DE LA PERSONA CON COLOSTOMÍA}

RESUMEN: Investigación exploratoria y descriptiva, realizada en 2013, cuyo objetivo fue elaborar un protocolo de asistencia de enfermería con los diagnósticos/ resultados e intervenciones de enfermería, relacionados a las necesidades psicosociales y psicoespirituales de la persona con colostomía. Fueron elaborados 47 diagnósticos/ resultados y 57 intervenciones de enfermería utilizando la Clasificación Internacional para la Práctica de Enfermería, organizados de acuerdo al referencial teórico de Wanda de Aguiar Horta. Se concluyó que la persona con colostomía se presenta fragilizada y demanda cuidados de enfermería específicos, individualizados, en razón de aflicciones, miedos y cambios en su estilo de vida. Esta clasificación de enfermería es de fácil utilización y su asociación con las necesidades humanas básicas permitió percibir la persona con colostomía de modo integral, para allá de las técnicas y procedimientos de enfermería.

DESCRIPTORES: Colostomía; Procesos de enfermería; Clasificación; Terminología; Teoría de enfermería.

\footnotetext{
*Artigo extraído da dissertação intitulada: Diagnósticos e intervenções de enfermagem para a pessoa com colostomia: uma tecnologia do cuidado. Universidade Federal do Espírito Santo, 2013.
} 


\section{INTRODUÇÃO}

As colostomias constituem parte de abordagens terapêuticas de trauma abdominal com lesão intestinal decorrente de acidentes por causas externas e também de doenças do intestino ou do ânus, como o câncer colorretal(1). São realizadas por meio de um procedimento cirúrgico, no qual ocorre exteriorização do cólon na parede abdominal (estoma), fornecendo assim um novo trajeto para eliminação do conteúdo intestinal $^{(2)}$.

Após uma cirurgia que resulta em colostomia, o paciente, ao se recuperar, defronta-se com modificações fisiológicas, psicossociais, psicoespirituais e com necessidades de adaptação para a convivência com o problema. Precisa iniciar uma nova fase da vida, incorporando em sua rotina diária o autocuidado, a manutenção de suas atividades sociais e interpessoais, os cuidados pós-operatórios, bem como compreender a própria doença, as alterações da imagem corporal, os sentimentos de luto, de perda, com reações e comportamentos diferentes dos que apresentava antes do estoma ${ }^{(3)}$.

Estão presentes na vida da pessoa com colostomia sentimentos como depressão; solidão; pensamentos suicidas; sentimentos de estigma; perda da autoestima e alteração da autoimagem; insegurança e medo do desconhecido $^{(4-5)}$.

É comum o paciente desenvolver um sentimento de autorrejeição, o que faz aumentar a sua insegurança. Esse período tornase mais difícil e dolorido com a rejeição social encontrada no seio da própria família, de quem deveria receber apoio e acolhimento para que a aceitação de sua condição fosse menos traumática ${ }^{(6)}$. As alterações das necessidades psicossociais e psicoespirituais exigem que Ihe sejam dispensados cuidados específicos para atender às suas demandas e colaborar na superação dessa nova condição de vida.

O relacionamento social fica muito difícil, não sentem vontade de sair de casa, limitam as atividades sociais por medo de "acidentes com a bolsa de colostomia", como o odor, a eliminação de gases ou o rompimento da bolsa ${ }^{(7)}$. As pessoas colostomizadas apontam o impacto que essa condição gera no seu estilo de vida e no de sua família, prejudicando os momentos de lazer. O lazer proporciona bem-estar físico e mental, é fonte de prazer e é importante para a constituição e manutenção das relações sociais, enquanto a falta de lazer altera a qualidade de $\operatorname{vida}^{(6)}$.

Vale ressaltar a importância do Processo de Enfermagem, uma vez que acrescenta qualidade ao cuidado prestado. Por meio do processo, o cuidado é focalizado na resposta do cliente, na forma como reage aos problemas de saúde, ao tratamento e às mudanças na vida diária, assegurando que as intervenções sejam elaboradas para o cliente, e não apenas para a doença ${ }^{(8-9)}$.

Para a realização de algumas das etapas do Processo de Enfermagem, como o diagnóstico, o resultado ea intervenção, são necessários o uso de sistemas de classificação. Neste estudo, optamos pela Classificação Internacional para a Prática de Enfermagem $\left(\mathrm{CIPE}^{\oplus}\right)$, por ser considerada como um sistema de linguagem unificada em enfermagem e ter sido reconhecida, desde 2008, como membro da família de classificações da Organização Mundial de Saúde ${ }^{(9-10)}$.

Tendo em vista essas questões e com o intuito de ajudar o colostomizado a enfrentar as dificuldades nesse período de adaptação, de modo a alcançar um bem-estar físico e psicológico, este estudo teve como objetivo elaborar um protocolo de assistência de enfermagem, contendo diagnósticos / resultados e intervenções de enfermagem, relacionados às necessidades psicossociais e psicoespirituais da pessoa com colostomia.

\section{MÉTODO}

Trata-se de um estudo exploratório-descritivo, que consistiu em três etapas, sendo a primeira etapa a revisão da literatura sobre cuidados de enfermagem e colostomia por meio de livrostexto da área de enfermagem, assistência cirúrgica e artigos científicos extraídos das bases: Literatura Latino-Americana e do Caribe em Ciências da Saúde (LILACS) e Medical Literature Analysis and Retrieval System Online (MEDLINE) com os descritores: "cuidados de enfermagem", "diagnóstico de enfermagem", "classificação", "colostomia", nos idiomas português, inglês e espanhol, publicados no período de 2000 a 2013. Foram excluídos dessa revisão trabalhos apresentados em congressos. Para direcionar a pesquisa, utilizou-se a questão norteadora: Quais os fenômenos e ações de enfermagem relacionadas à pessoa com colostomia? Entendese por esses fenômenos e ações os aspectos de saúde relevantes para a enfermagem e os 
cuidados que os enfermeiros executam no que diz respeito às necessidades humanas, para alcançar determinados resultados.

Foram encontrados 182 artigos no LILACS e 252 no MEDLINE. Desse total de 434 artigos, 47 foram selecionados e utilizados para consulta, visto que alguns eram repetidos e outros não respondiam à questão norteadora deste estudo.

A segunda etapa consistiu do mapeamento dos termos identificados na revisão de literatura com os termos do Modelo de Sete Eixos da CIPE $^{\circledR(11)}$. A terceira etapa foi a elaboração do protocolo de enfermagem contendo os diagnósticos / resultados e intervenções de enfermagem relacionados à assistência à pessoa com colostomia, organizados a partir das necessidades humanas básicas alteradas ${ }^{(12)}$. Para a construção dos diagnósticos / resultados de enfermagem deve-se incluir, obrigatoriamente, um termo do eixo Foco e um termo do eixo Julgamento e termos adicionais dos outros eixos, conforme a necessidade; para elaborar as intervenções de enfermagem recomendase incluir um termo do eixo Ação e um do eixo Alvo, sendo que esses termos podem pertencer a qualquer um dos eixos, exceto ao do Julgamento ${ }^{(10)}$.
Este estudo foi aprovado pelo Comitê de Ética em Pesquisa do Centro de Ciências da Saúde da Universidade Federal do Espírito Santo, sob número 09167813.1.0000.5060, em 22 de fevereiro de 2013.

\section{RESULTADOS}

Considerando as necessidades humanas básicas psicossociais e espirituais alteradas, específicas para o paciente com colostomia, foram elaborados 47 diagnósticos / resultados de enfermagem e 57 intervenções de enfermagem, utilizando a $\mathrm{CIPE}^{\circledR}$ que estão apresentados no Quadro 1. Para isso, foram utilizados 14 termos do eixo "Foco", 09 termos do eixo "Julgamento" e 01 termo do eixo "Localização". Elencaram-se os termos do eixo "Foco": socialização, divertirse, ansiedade, insegurança, medo, aceitação do estado de saúde, autoimagem negativa, autoestima baixa, vergonha, conhecimento, regime de cuidados com o estoma, autocuidado, recursos materiais terapêuticos e angustia espiritual. Os termos do eixo "Julgamento" utilizados foram: risco, diminuído, eficaz/ adequado, prejudicado, melhorado, baixo/ falta/insuficiente/déficit, positivo, negativo, disposição. O termo do eixo "Localização" utilizado foi: colostomia.

Quadro 1 - Protocolo de assistência de enfermagem para as necessidades psicossociais e psicoespirituais da pessoa com colostomia. Vitória, ES, Brasil, 2014

\section{INTRODUCÃO}

Pacientes estomizados apresentam insegurança e vergonha devido aos odores e gases da colostomia; o cuidado com a bolsa coletora interfere no relacionamento social; referem rejeição quanto à imagem corporal e falta de orientação sobre a cirurgia e os cuidados a serem realizados diariamente com a colostomia(7,13-14).

\section{OBJETIVO}

Padronizar as condutas clínicas de enfermagem para as alterações psicossociais e espirituais da pessoa com colostomia.

\section{ATIVIDADES ESSENCIAIS}

Realizar o Processo de Enfermagem e registrá-lo utilizando a classificação CIPE ${ }^{\circledR}$ por meio de diagnósticos, resultados e intervenções de enfermagem para as necessidades psicossociais e psicoespirituais.

\section{DIAGNÓSTICOS/RESULTADOS/INTERVENÇÕES}

Necessidade gregária

Necessidade de recreação e lazer

Necessidade de amor e aceitação

Necessidade de segurança emocional

Necessidade de educação para a saúde e aprendizagem

Necessidade de autoestima, autoconfiança e autorrespeito

Necessidade de autorrealização

Necessidade de garantia de acesso à tecnologia

Necessidade de religiosidade e espiritualidade 
Quadro 2 - Diagnósticos, resultados e intervenções de enfermagem para as necessidades gregária; recreação e lazer; amor e aceitação da pessoa com colostomia. Vitória, ES, Brasil, 2014

\begin{tabular}{lc} 
DIAGNÓSTICOS/RESULTADOS & INTERVENÇÕES \\
\hline Necessidade gregária & \\
\hline Socialização diminuída; & Encorajar participação em atividades sociais; \\
Risco de socialização diminuída; & Encorajar participação em grupos de apoio, como a Associação \\
Socialização adequada. & Brasileira de Ostomizados; \\
& Orientar quanto à importância do convívio social; \\
& Encorajar a realização de autoirrigação intestinal; \\
& Encorajar o retorno às atividades de rotina; \\
& Encorajar o retorno ao convívio social. \\
\hline
\end{tabular}

Necessidade de recreação e lazer

Lazer diminuído; $\quad$ Orientar quanto à importância do lazer;

Risco de lazer diminuído; $\quad$ Orientar quanto à importância do convívio social;

Divertir-se adequado. Identificar as preferências de recreação do paciente;

Estimular a recreação e o lazer;

Encorajar a recreação de acordo com as limitações do ostomizado;

Estimular a recreação com a família;

Estimular a recreação com grupos de ostomizados.

\section{Necessidade de amor e aceitação}

Aceitação do estado de saúde

Risco de aceitação do estado de saúde diminuída;

Aceitação do estado de saúde diminuída;

Aceitação do estado de saúde

melhorada.
Encorajar participação em atividades sociais;

Encorajar participação em grupos de apoio, como a Associação

Brasileira de Ostomizados;

Investigar a aceitação do paciente quanto à colostomia;

Investigar a aceitação da família quanto à colostomia.

Quadro 3 - Diagnósticos, resultados e intervenções de enfermagem para as necessidades de segurança emocional; educação para a saúde e aprendizagem da pessoa com colostomia. Vitória, ES, Brasil, 2014

\section{DIAGNÓSTICOS/RESULTADOS}

\section{Necessidade de segurança emocional}

Ansiedade;

Risco de ansiedade;

Ansiedade melhorada;

Insegurança;

Risco de insegurança;

Insegurança melhorada;

Medo;

Risco de medo;

Medo melhorado.

\section{INTERVENÇÕES}

Identificar situações que causam ansiedade;

Identificar situações que causam medo;

Identificar situações que causam insegurança;

Encorajar o paciente a controlar a ansiedade;

Encorajar o paciente a falar sobre seu medo;

Estimular o enfrentamento;

Oferecer apoio psicológico;

Responder a dúvidas;

Avaliar atitudes em relação à colostomia;

Avaliar enfrentamento em relação à colostomia;

Encorajar participação em grupos de apoio, como a

Associação Brasileira de Ostomizados.

\section{Necessidade de educação para a saúde e aprendizagem}

Conhecimento insuficiente sobre colostomia; Encorajar participação em grupos de apoio, como a Risco de conhecimento insuficiente sobre Associação Brasileira de Ostomizados;

colostomia;

Falta de conhecimento sobre colostomia;

Responder as dúvidas relacionadas aos cuidados com o estoma;

Risco de falta de conhecimento sobre colostomia;

Conhecimento adequado sobre colostomia;

Regime de cuidados prejudicado com o estoma;

Risco de regime de cuidados prejudicado com o estoma;

Regime de cuidados eficaz com o estoma.
Orientar o autocuidado com a colostomia;

Monitorar o autocuidado com a colostomia;

Encaminhar ao Programa de Ostomizados;

Orientar os cuidados com a pele ao redor da colostomia;

Orientar a autoirrigação da colostomia;

Monitorar a autoirrigação da colostomia. 
Quadro 4 - Diagnósticos, resultados e intervenções de enfermagem para as necessidades de autoestima, autoconfiança e autorrespeito; autorrealização; e garantia de acesso à tecnologia da pessoa com colostomia. Vitória, ES, Brasil, 2014

\section{DIAGNÓSTICOS/RESULTADOS}

\section{INTERVENCÕES}

\section{Necessidade de autoestima, autoconfiança e autorrespeito}

Autoimagem negativa;

Risco de autoimagem negativa;

Autoimagem positiva;

Estimular o paciente a identificar atributos pessoais positivos;

Baixa autoestima;

Risco de baixa autoestima;

Encorajar pensamentos positivos;

Encorajar o paciente a se arrumar;

Autoestima positiva;

Vergonha da colostomia;

Estimular a recreação com a família;

Estimular a recreação com grupos de ostomizados;

Risco de vergonha da colostomia;

Vergonha melhorada da colostomia;

Vergonha ausente da colostomia.

\section{Necessidade de autorrealização}

Déficit de autocuidado com a colostomia;

Risco de déficit de autocuidado com a colostomia;

Autocuidado eficaz com a colostomia,

Disposição para o autocuidado eficaz com a colostomia.
Estimular o autocuidado com a colostomia;

Identificar adaptações necessárias para o autocuidado;

Encorajar participação em grupos de apoio, como a Associação Brasileira de Ostomizados;

Responder as dúvidas relacionadas aos cuidados com o estoma;

Orientar o autocuidado com a colostomia;

Monitorar o autocuidado com a colostomia;

Encaminhar ao Programa de Ostomizados;

Orientar os cuidados com a pele ao redor da colostomia.

\section{Necessidade de garantia de acesso à tecnologia}

Recursos materiais terapêuticos inadequados (bolsa Reforçar a importância da participação na Associação de colostomia, protetores de pele, outros);

Recursos materiais terapêuticos adequados (bolsa de colostomia, protetores de pele, outros). de Ostomizados;

Orientar quanto ao direito de ganhar a bolsa de colostomia;

Orientar quanto aos tipos de bolsa de colostomia.

Quadro 5 - Diagnósticos, resultados e intervenções de enfermagem para as necessidades psicoespirituais da pessoa com colostomia. Vitória, ES, Brasil, 2014

\section{DIAGNÓSTICOS/RESULTADOS}

\section{INTERVENCÕES}

\section{Necessidade de religiosidade e espiritualidade}

Angústia espiritual;

Risco de angústia espiritual;

Angústia espiritual diminuída;

Angústia espiritual melhorada.
Apoiar práticas espirituais da pessoa e da família;

Encaminhar ao líder espiritual para atendimento à pessoa e à família;

Proporcionar ambiente que favoreça a expressão de religiosidade e espiritualidade;

Promover o bem-estar do paciente.

\section{DISCUSSÃO}

A ocorrência de uma colostomia implica um sistema complexo de mudanças no cotidiano da pessoa. É uma fase crítica que necessita de análise e reflexões, tendo em vista as experiências construídas pela pessoa ao longo de sua vida ${ }^{(8)}$.

\section{Um paciente com colostomia vivencia}

sentimentos diversos, como medo, sofrimento, dor emocional, ansiedade. Portanto, necessita de um cuidado sensível que o torne forte e capaz de enfrentar desafios e limitações ${ }^{(15)}$. Para superar os sentimentos de perda, negação, revolta, desesperança que o acometem, é fundamental o apoio e o estímulo de pessoas significativas bem como a ajuda do enfermeiro, cujo papel é importante em todo o processo até 
sua reinserção social ${ }^{(13,16)}$.

Além de ter que aprender os cuidados com a colostomia, o colostomizado necessita também de orientações quanto à provisão dos dispositivos ou sistemas coletores, que são compostos por placas e bolsas; adequações de vestuário para que não apertem a bolsa, evitando que se desloque; adaptações alimentares para minimizar gases e odores intestinais durante as interações sociais ${ }^{(14)}$.

A perda de controle da eliminação de fezes e gases pode levar o paciente ao isolamento psicossocial, à baixa autoestima, a sentimentos de incapacidade na gestão da nova condição em que se encontra. $\mathrm{O}$ fato de necessitar de condições especiais para troca dos dispositivos acaba por restringir o convívio social e até mesmo viagens. Portanto, uma rede de apoio de familiares, amigos, grupos sociais, grupos de autoajuda são importantes para melhorar a aceitação e adaptação do colostomizado ${ }^{(16)}$.

Uma higiene eficaz e cuidados adequados com a bolsa minimizam os odores e o risco de acidentes, melhorando a autoconfiança, além de diminuir a ansiedade e os medos do colostomizado facilitando assim sua socialização ${ }^{(17)}$. A realização da auto-irrigação da colostomia provoca um efeito positivo sobre a qualidade de vida do colostomizado, devido ao controle que a maioria das pessoas consegue obter na eliminação de fezes e gases pelo estoma, o que lhes proporciona segurança e conforto nas relações sociais ${ }^{(18)}$.

A participação em programas de estomizados e em grupos de autoajuda colabora de maneira positiva, diminuindo o sentimento de solidão, visto que proporciona a troca de experiências e a percepção de sentir-se semelhante a outros com os mesmos problemas. Isso fortalece sua autoconfiança e tem grande valor terapêutico ${ }^{(19)}$.

É importante enfatizar também a contribuição do lazer como fonte não só de prazer, mas também de manutenção das relações sociais, para o bem-estar físico e psíquico do paciente com colostomia, pois tudo o que prejudica o lazer altera a qualidade de vida do ser humano(6).

A família e os amigos formam uma rede de cumplicidade e de apoio imprescindível para que o colostomizado supere os problemas e encontre as soluções adequadas para essa nova etapa de sua vida ${ }^{(20)}$. Os efeitos positivos causados pela religiosidade/espiritualidade traduzem-se em mais força para a superação dessa fase da vida, causando bem-estar e satisfação ${ }^{(16,19)}$.

Todas essas transformações ocorridas na vida da pessoa com colostomia necessitam de tempo para que ela aceite a sua nova imagem corporal e aprenda a se autocuidar. Para tanto, a educação em saúde é primordial e indispensável ${ }^{(4)}$.

O enfermeiro tem um papel social importante e influencia na forma como essa pessoa com colostomia vai encarar as mudanças necessárias para sua adaptação. Portanto, reforça-se a necessidade de utilização do Processo de Enfermagem, visto que amplia a atuação desse profissional, estimulando seu raciocínio crítico e sua autonomia ao dispensar cuidados e orientações de forma a atender a individualidade do ser humano(21).

O Processo de Enfermagem sistematiza a prática profissional do enfermeiro, direcionando seu modo de pensar e sua tomada de decisões, favorecendo e organizando o cuidado prestado comointuitodealcançaros resultadosnecessários para atender as necessidades alteradas ${ }^{(8-9)}$. Os enfermeiros consideram a sistematização da assistência muito importante, referindo que melhora a qualidade da assistência, promove autonomia e permite a unificação da linguagem, mesmo que a maioria dos enfermeiros $(70 \%)$ não utilizem os diagnósticos em sua prática profissional(22).

Estudos apontam que a CIPE ${ }^{\circledR}$, uma linguagem padronizada, facilita a comunicação entre os enfermeiros e os profissionais de saúde, aperfeiçoa os cuidados prestados, padroniza a documentação e o planejamento do cuidado prestado por meio das intervenções de enfermagem ${ }^{(9-10,23)}$.

\section{CONCLUSÃO}

A pessoa com colostomia apresenta-se fragilizada, podendo apresentar alterações de ordem psicológica, emocional e social. Portanto, necessita de acolhimento e apoio para superar essa fase de adaptação. A reinserção social constitui um desafio para o enfermeiro que precisa encorajar o colostomizado e sua família a aceitar e conviver com o estoma.

Foram elaborados 47 diagnósticos e 57 intervenções de enfermagem para a pessoa com colostomia relacionados às necessidades psicossociais e espirituais. Destaca-se, dentre as intervenções elaboradas, a importância 
do incentivo ao paciente na participação dos grupos de autoajuda e na Associação Brasileira de Ostomizados. É importante enfatizar o autocuidado, estimulando a participação e o envolvimento efetivo do colostomizado, de modo a contribuir para a sua reabilitação e para a superação das dificuldades encontradas.

Estudos como este podem contribuir para o planejamento adequado do cuidado que deve ser prestado a esse paciente, uma vez que o enfermeiro desempenha papel importante na orientação e assistência a ele e à sua família. Também reforça a importância da utilização de uma linguagem padronizada na elaboração de diagnósticos e intervenções, o que pode ser conseguido com o uso da CIPE ${ }^{\circledR}$.

No decorrer desse estudo percebeu-se que a CIPE $^{\circledR}$ é de fácil utilização, pois seus termos são relacionados com a prática clínica. A identificação das necessidades humanas básicas alteradas e o desenvolvimento de um raciocínio clínico para organizar os diagnósticos de enfermagem possibilitou uma percepção mais ampla do cliente. A associação das necessidades humanas básicas aos diagnósticos / resultados e intervenções de enfermagem da CIPE ${ }^{\circledR}$ permitiu perceber a pessoa com colostomia de maneira integral para além das técnicas e procedimentos de enfermagem.

Esta pesquisa possibilitou a elaboração de um protocolo de assistência de enfermagem para a pessoa com colostomia, e contribuiu para a produção de novas tecnologias na área da enfermagem, pois a CIPE $^{\circledR}$ é um instrumental tecnológico que visa padronizar a linguagem de enfermagem para uso nos sistemas de informação em saúde e documentação eletrônica.

Espera-se incentivar os enfermeiros a realizar o Processo de Enfermagem em todas as etapas e a utilizar a $\mathrm{CIPE}^{\circledR}$ para sistematizar a assistência prestada, contribuindo para a melhoria da qualidade do cuidado prestado por esse profissional.

\section{REFERÊNCIAS}

1. Malagutti W, Kakihara CT. Curativos, estomias e dermatologia: uma abordagem multiprofissional. São Paulo: Martinari; 2011.

2. Silva RCL, Figueiredo NMA, Meireles IB. Feridas: fundamentos e atualizações em enfermagem. São Caetano do Sul: Yends; 2007.
3. Costa SPR. Perfil de qualidade de vida dos portadores de colostomia [dissertação]. João Pessoa (PB): Universidade Federal da Paraíba; 2007.

4. Batista MRFF, Rocha FCV, da Silva DMG, da Silva Júnior FJGS . Autoimagem de clientes com colostomia em relação à bolsa coletora. Rev Bras Enferm. 2011;64(6):1043-7.

5. Virginio NA, Nobrega MML. Validação de instrumento de coleta de dados de enfermagem para clientes adultos hospitalizados. Rev Bras Enferm. 2004;57(1):53-6.

6. Bellato R, Maruyama SAT, Silva CM, Castro P. A condição crônica ostomia e as repercussões que traz para a vida da pessoa e sua família. Cienc. Cuid. Saude. [Internet] 2007;6(1) [acesso 25 set 2012]. Disponível: http://periodicos.uem.br/ojs/index.php/ CiencCuidSaude/article/view/4971

7. Santos GS, Leal SMC, Vargas MA. A closer look at women who underwent colostomy surgery: a qualitative exploratory-descriptive study. OBJN. [Internet] 2006;5(1) [acesso em 15 set 2012]. Disponível: http://www.objnursing.uff.br/index.php/nursing/ article/view/82

8. GarciaTR, Nóbrega MML.Processo de enfermagem: da teoria à prática assistencial e de pesquisa. Esc. Anna Nery. 2009;13(1):188-93.

9. Barra DCC, Dal Sasso GTM. Processo de enfermagem conforme a Classificação Internacional para as Práticas de Enfermagem: Uma Revisão Integrativa. Texto Contexto Enferm. 2012;21(2) [acesso em 25 ago 2013] Disponível: http://dx.doi.org/10.1590/ S0104-07072012000200024

10. Primo CC, Cabral LA, Cuquetto SC, Laignier MR, Lima EFA, Leite FMC. Diagnósticos de enfermagem relacionados ao fenômeno amamentação exclusiva. Cogitare enferm [Internet] 2013;18(2) [acesso em 25 out 2013] Disponível: http://dx.doi.org/10.5380/ ce.v18i2.28475

11. Conselho Internacional de Enfermeiros. CIPE, Versão 2013: Classificação Internacional para a Prática de Enfermagem [acesso 3 jul 2013]. Disponível: http://www.icn.ch/images/stories/documents/pillars/ Practice/icnp/translations/icnp-Brazil-Portuguese_ translation.pdf

12. Garcia TR, Cubas MR. Diagnósticos, intervenções e resultados de enfermagem: subsídios para a sistematização da prática profissional. Rio de Janeiro: Elsevier; 2012.

13. Coelho AR, Santos FS, Poggetto MTD. A estomia mudando a vida: enfrentar para viver. Rev Min Enferm. 
[Internet] 2013;17(2) [acesso em 25 set 2013] Disponível: http://dx.doi.org/10.5935/1415-2762.20130021

14. Maruyama SAT, Zago MMF. O processo de adoecer do portador de colostomia por câncer. Rev. Latino-am. Enferm. 2005;13(2):216-22.

15. Delavechia RP, Terra MG, Noal HC, Padoin SMM, Lacchin AJB, Silva MEN. A percepção de si como serestomizado: um estudo fenomenológico. Rev. Enferm. UERJ. 2010;18(2):223-28.

16. Sousa CF, Brito DC, Branco MZPC. Depois da colostomia... vivências das pessoas portadoras. Enferm. Foco. 2012;3(1):12-5.

17. Carpenito-Moyet LJ. Planos de cuidados de enfermagem e documentação: diagnósticos de enfermagem e problemas colaborativos. Porto Alegre: Artmed; 2011.

18. Cesaretti IUR, Santos VLCG, Schiftan SS, Vianna LAC. Irrigação da colostomia: revisão acerca de alguns aspectos técnicos. Acta paul. enferm. 2008;21(2):33844.

19. Santana J, Dutra B, Tameirão M, Silva P, Moura I, Campos A. O significado de ser colostomizado e participar de um programa de atendimento ao ostomizado. Cogitare enferm. [Internet] 2010;15(4) [acesso em 15 jul 2013] Disponível: http://dx.doi. org/10.5380/ce.v15i4.20358

20. Dázio EMR, Sonobe HM, Zago MMF. Os sentidos de ser homem com estoma intestinal por câncer colorretal: uma abordagem na antropologia das masculinidades. Rev. Latino-am. Enferm. [Internet] 2009;17(45) [acesso em 01 jul 12] Disponível: http:// dx.doi.org/10.1590/S0104-11692009000500011

21. Menezes SRT, Priel MR, Pereira LL. Autonomia e vulnerabilidade do enfermeiro na prática da sistematização da assistência de enfermagem. Rev. Esc. Enferm. USP. [Internet] 2011;45(4) [acesso em 15 jul 2013] Disponível: http://dx.doi.org/10.1590/S008062342011000400023

22. Silva EGC, Oliveira VC, Neves GBC, Guimarães TMR. O conhecimento do enfermeiro sobre a sistematização da assistência de enfermagem: da teoria à prática. Rev. Esc. Enferm. USP. 2011;45(6) [acesso em 17 jul 2013]. Disponível: http://dx.doi.org/10.1590/ S0080-62342011000600015

23. Primo CC, Lima EFA, Leite FMC, Cruz MAS, Ferreira FM. Nursing diagnosis related to the adverse effects of radiotherapy. Rev. Min. Enferm. [Internet] 2013;17(4) [acesso em 05 jan 2014] Disponível: http://www.dx.doi. org/10.5935/1415-2762.20130068 